\title{
Knowledge, attitudes, and influencing factors of cancer patients toward approving advance directives in China
}

\author{
Ru-jun Zheng ${ }^{1,2} \cdot$ Yan Fu $^{2}$ • Qiu-fen Xiang ${ }^{2} \cdot$ Mei Yang ${ }^{3} \cdot$ Lin Chen $^{4} \cdot$ Ying-kang Shi ${ }^{5}$. \\ Chun-hua $\mathrm{Yu}^{2}$. Jun-ying $\mathrm{Li}^{2}$
}

Received: 5 January 2016 / Accepted: 17 April 2016/Published online: 21 May 2016

(C) The Author(s) 2016. This article is published with open access at Springerlink.com

\begin{abstract}
Purpose Many cancer patients do not have advance directives (ADs), which may lead to unwanted excessive or aggressive care when patients have lost decision-making capacity. The aim of this study was to investigate knowledge and attitudes of approving ADs and explore factors associated with willing to designate ADs among cancer patients in China.

Methods We conducted semi-structured interview method investigating 753 in-patients with cancer in two cancer centers. Results Of those subjects, none of the cancer patients had an AD. Only $22.4 \%$ (118 of 526) approved ADs. Comparing with the disapproved ADs group, the approved ADs group were more likely to discuss the $\mathrm{AD}$ with oncologist or nurse $\left(\chi^{2}=180.4\right.$, $p<0.001)$ in the cancer center $\left(\chi^{2}=244.1, p<0.001\right)$, and they chose more comfort care $\left(\chi^{2}=18.8, p<0.001\right)$. Most of cancer patients in the two groups wanted to die at home $(72.8 \%$,
\end{abstract}

Yan $\mathrm{Fu}$ contributed equally to this work.

Chun-hua Yu

chunhua1995@126.com

Jun-ying Li

lijunying2005@sina.com

1 West China Nursing School and Business School, Sichuan University, Chengdu 610041, People's Republic of China

2 Department of Thoracic Cancer and Cancer Research Center, West China Hospital, Sichuan University, Chengdu 610041, People's Republic of China

3 Cancer Research Center, Tumor Hospital of Xinjiang, Xinjiang Medical University, Urumuqi 830000, People's Republic of China

4 Lung Cancer Center, West China Hospital, Sichuan University, Chengdu 610041, People's Republic of China

5 Institute of Hospital Administration,West China Hospital, Sichuan University, Chengdu 610041, People's Republic of China
$73.7 \%$, respectively). The older patients (OR, 1.04, $95 \% \mathrm{CI}$, $1.02-1.07, p=0.001$ ), female (OR, 0.55, $95 \%$ CI, 0.35-0.88, $p=0.013)$, with higher education levels (OR, 3.38, $95 \% \mathrm{CI}$, $1.92-5.96, p<0.001$ ), with religious beliefs (OR, 2.91, $95 \% \mathrm{CI}$, $1.71-4.94, p<0.001$ ), and with higher scores of ECOG (OR, $1.46,95 \%$ CI, $1.17-1.82, p=0.001)$ were associated with desiring for ADs.

Conclusions Our findings indicate that there was a dearth of knowledge and different attitudes toward approving ADs among cancer patients, and some factors of demographic and clinical characteristics influenced their willing to designate ADs. This research highlights the importance of propagandizing the ADs to the public, especially to the patients, and further discussing with them when the time is ripe.

Keywords Knowledge $\cdot$ Attitude $\cdot$ Factor $\cdot$ Advance directive $\cdot$ Cancer patient

\section{Introduction}

Over the past 3 decades, there has been more attention paid to raising patients' rights of making medical decisions regarding their own treatment [1-4]. ADs is a process of discussion and a formalized document with patients and their caregivers about their wishes for treatment and care planning when they become unable to make medical decision because of illness or incapacity. In several countries, ADs has been legalized since the California Natural Death Act was introduced in 1976 [4-8]. The Patient Self-Determination Act (PSDA) requires that Medicare and Medicaid providers inform all patients of their rights to complete ADs, to make their health care preferences [5]. In China, we do not strictly distinguish "Advance Directives," "Living Wills," and "Advance Care Planning" in practice [9]. The biggest barrier to the development of $\mathrm{ADs}$ is that some people consider 
withholding life-sustaining treatments by ADs for a terminally ill patient to be an act of passive euthanasia, and the Chinese government is firm in its stand against euthanasia (The criminal law of the People's Republic of China, 2015). However, in practice, the family members often withdraw life-sustaining treatments for a terminally ill patient [10].

End-of-life (EOL) issues such as advance care planning, palliative care, place of death, and the family roles are valuable to the cancer patients [11-14]. Studies have found written ADs were associated with reduced aggressive medical treatment [15-18], decreased rate of hospitalization [18], and lower hospital charges [17, 19]. Completing ADs keeps caregivers out of the difficult situation of having to guess what kind of care or treatment the care recipients accept or refuse. This is also the reason that the families and caregivers think that ADs are valuable. So, it is necessary for the cancer patients to have an AD.

The rate of having an $\mathrm{AD}$ among subjects is low in most of researches. Previous studies indicate that completion of ADs was 15 to $41 \%$ among the cancer patients [11, 20, 21]; 9 to $70 \%$ among older adults $[2,5,16,22] ; 15$ to $30 \%$ among general population [23]; $7 \%$ among hemodialysis patients [24]; $6 \%$ among hematological malignancy patients [25]. Dow reported that $63 \%$ of oncology patients did not know what an $\mathrm{AD}$ was [11], and Ewer doubted about the purpose and role of ADs [26]. This may explain that a large number of subjects refused to use ADs.

Recent studies showed that use of ADs was associated with sociodemographic characteristics [2, 21, 27-30]. Previous studies have found that persons with ADs documents were more likely to die at home than in a hospital [2, 18]. Absence of ADs can lead to unwanted aggressive medical care while patients lacking decision-making capacity [2, 31]. Tan et al. reported that older people and having cancer therapy were more likely to have an $\mathrm{AD}$ [21]. It was reported that older age $[11,30]$ and health insurance $[30]$ were associated with a greater likelihood of ADs completion. Periyakoil et al. reported that gender, ethnicity, and sub-specialty were the critical influences on the attitudes toward ADs [27]. Holley et al. reported that gender, age, race, religion, and education were significantly associated with ADs completion [28]. However, in Molloy et al. study, no significant difference was found between ADs completion and gender [16]. Therefore, there was no unanimous conclusion between ADs and sociodemographic characteristics.

Little is known about the association between demographic characteristics and willing to designate ADs among cancer patients. Despite the advantage of the ADs, only 10,000 Chinese completed the document of ADs in 2014 [32]. To the best of our knowledge, no reports were found of Chinese cancer patients' completion rate of ADs and the knowledge and attitudes of approving ADs. The aim of this study was to investigate knowledge and attitudes of approving ADs and explore factors associated with willing to designate ADs among cancer patients in China. It can offer data support to clinical decision-making and patient education about ADs.

\section{Methods}

\section{Study sample}

Two departments of oncology from two university hospitals were involved in this study. Of 753 consecutive cancer patients admitted to the two hospitals inpatient service between June 1, 2015 and November 31, 2015, 526 (69 \%) were recruited into our study. Of the 227 patients who did not participate in the study, 107 were not interested in the research and they refused our invitation, 57 were not be allowed because of their illness, 38 failed to complete the questionnaire or interview, 21 were minority who spoke the minority languages and they did not know Chinese simplified characters, and 4 were under 18 years. All patients knew their diagnosis of cancer before participation.

\section{Questionnaire}

We used a questionnaire to assess cancer patients' knowledge and attitudes toward ADs (Table 1). This questionnaire was based on previous studies, and most of the questions were adopted in the questionnaire after modification to fit local culture and traditions [2, 3, 11, 28, 33-35]. The questionnaire comprised 25 questions, 11 were designed to gather demographic clinical characteristics (age, gender, marital status, education, religion, health insurance, primary tumor site, etc., and without signature or ID card), 14 were used to collect information regarding the knowledge and attitudes of cancer patients. In this questionnaire, patients should answer seven open-ended questions depending on their knowledge and experience. Before formal interview, the questionnaire was pilot

Table 1 Main questions in the questionnaire

Do you know about ADs, and how do you know the ADs?

Do you know what an AD is, and what is the purpose or meaning of ADs?

Who do you prefer to discuss ADs, and why do you choose that? (The two questions will examine informations about harmonious family relationship.)

$\square$ Spouse $\square$ Parent $\square$ Child $\square$ Oncologist or nurse

Where do you prefer to discuss ADs, and why do you choose that? $\square$ Home $\square$ Nursing home $\square$ Hospice $\square$ Cancer center

When do you prefer to discuss ADs, and why do you choose that? $\square$ Diagnosis immediately $\square$ Cancer advanced $\square$ Terminally ill

Which kind of care do you need, and why do you choose that? $\square$ Comfort care only $\square$ Limited care $\square$ All care possible

Where do you want to die, and why do you choose that? $\square$ Home $\square$ Nursing home $\square$ Hospital

$A D s$ advance directives 
tested with 30 cancer patients in order to revise it for well understanding and clarity. We also provided the specified space for the interview.

\section{Data collection}

We developed a semi-structured interview for the study to collect demographic data and assess patient knowledge and attitudes toward ADs. The information regarding knowledge and attitudes included (Table 1): (1) whether and how to know ADs; (2) what is an $\mathrm{AD}$, and what is the purpose or meaning of ADs; (3) the prefer person, place and time to discuss ADs topics, and why they choose those; (4) which care the patients need, and which place they want to die, and why they choose those. Each of hospital has one clinical nurse to practice the interview that all participants consented. All the patients were told information explaining the research and asked not to share their responses with other patients. The two interviewers recorded answers to the questions without any attempt to impact on patients' decisions. Then, data were analyzed by an independent researcher who was not related to the data collection. The Ethics Committee of the West China Hospital approved the study. All the information in our study are confidential and available only by the aim of research.

\section{Data analysis}

The patient demographic data and knowledge toward ADs with statistical description. The chi-squared test was employed to find significant difference in attitudes of ADs between the two groups. The binary logistic regression analysis was used to estimate the odds ratio for each independent variable, to assess which of the factors associated with willing to designate ADs. We used SPSS statistic software (version $18.0)$ to analysis the data. We defined that $p$ value of less than 0.05 will be statistically significant ( $p$ value two-sided).

\section{Results}

\section{Patients characteristics}

The mean age of the cancer patients was 53.6 years (range, 20 to 81 years), 308 (58.6\%) were male, 418 (79.5\%) were married, 438 (83.3\%) owned health insurance, 134 (25.5\%) completed college, 398 (75.7\%) lived with family members, $94(17.9 \%)$ had religion, and $229(43.5 \%)$ had cancer metastasis. The annual income of the most of subjects $(88.2 \%)$ was under $100 \mathrm{~K}$. In terms of ECOG performance status, $208(39.5 \%)$ were 0 scores, $147(28.0 \%)$ were 1 scores, 123 $(23.4 \%)$ were 2 scores, and $48(9.1 \%)$ were 3 scores (Table 2).
Table 2 Characteristics of 526 study subjects

\begin{tabular}{|c|c|c|}
\hline Variables & No. & Percentage \\
\hline Mean age \pm SD & $53.6 \pm 13.2$ & \\
\hline \multicolumn{3}{|l|}{ Gender } \\
\hline Male & 308 & 58.6 \\
\hline Female & 218 & 41.4 \\
\hline \multicolumn{3}{|l|}{ Marital status } \\
\hline Married & 418 & 79.5 \\
\hline Single or divorced & 108 & 20.5 \\
\hline \multicolumn{3}{|l|}{ Income (annual) } \\
\hline$\leq 10 \mathrm{k}$ & 96 & 18.3 \\
\hline $10-50 \mathrm{k}$ & 168 & 31.9 \\
\hline $50-100 \mathrm{k}$ & 200 & 38.0 \\
\hline$\geq 100 \mathrm{k}$ & 62 & 11.8 \\
\hline \multicolumn{3}{|l|}{ Health insurance } \\
\hline Own & 438 & 83.3 \\
\hline Not own & 88 & 16.7 \\
\hline \multicolumn{3}{|l|}{ Education } \\
\hline Complete college & 134 & 25.5 \\
\hline Not complete college & 392 & 74.5 \\
\hline \multicolumn{3}{|l|}{ Primary tumor site } \\
\hline Head and neck neoplasm & 122 & 23.2 \\
\hline Thoracic neoplasms & 134 & 25.5 \\
\hline Abdominal neoplasms & 140 & 26.6 \\
\hline Limbs neoplasms & 130 & 24.7 \\
\hline \multicolumn{3}{|l|}{ Metastases } \\
\hline Yes & 229 & 43.5 \\
\hline No & 297 & 56.5 \\
\hline \multicolumn{3}{|l|}{ Living situation } \\
\hline With family members & 398 & 75.7 \\
\hline With others & 76 & 14.4 \\
\hline Alone & 52 & 9.9 \\
\hline \multicolumn{3}{|l|}{ Religiousness } \\
\hline Religious & 432 & 82.1 \\
\hline Nonreligious & 94 & 17.9 \\
\hline \multicolumn{3}{|l|}{ ECOG performance status } \\
\hline 0 & 208 & 39.5 \\
\hline 1 & 147 & 28.0 \\
\hline 2 & 123 & 23.4 \\
\hline 3 & 48 & 9.1 \\
\hline
\end{tabular}

$S D$ standard deviation, ECOG Eastern Cooperative Oncology Group

\section{Knowledge of ADs}

All of 526 cancer patients reported that they had not completed an AD. Ninety-seven percent (510 of 526) of the cancer patients had not heard of the terminology "advance directive." None of patients can define an AD accurately or know its purpose before our interviewers told them; however, $46.6 \%$ (245 of 526) were interested in ADs (data not displayed). 


\section{Attitudes of ADs}

Of the 526 cancer patients interviewed, $22.4 \%$ (118 of 526) approved the ADs, $77.6 \%$ (408 of 526) did not. 55.9\% (66 of 118) of respondents in the approved ADs group preferred to discuss the ADs topic with oncologist or nurse versus $4.7 \%$ (19 of 408) of those in the disapproved ADs group $\left(\chi^{2}=180.4, p<0.001\right)$. Seventy-five patients in the approved ADs group were more willing (63.3 versus $3.9 \%, p<0.001$ ) to discuss about ADs in the place of cancer center than those in the disapproved ADs group. The respondents who approved the ADs $(77.1 \%)$ were more likely to choose comfort care near the EOL compared with $56.4 \%$ of those in the disapproved ADs group $\left(\chi^{2}=18.8, p<0.001\right)$. In both of the two groups, a small number of cancer patients (15.9 and $18.6 \%$, respectively) wanted to discuss the ADs issue at the time of cancer diagnosis immediately, and most of respondents (72.8 and $73.7 \%$, respectively) would like to pass away at home (Table 3 ).

\section{Influencing factors toward ADs}

The binary logistic regression analysis revealed that the older patients (OR, 1.04, $95 \% \mathrm{CI}, 1.02-1.07, p=0.001)$, female (OR, 0.55, $95 \% \mathrm{CI}, 0.35-0.88, p=0.013)$, with higher education levels (OR, 3.38, $95 \%$ CI, 1.92-5.96, $p<0.001)$, with religious beliefs (OR, 2.91, 95\% CI, 1.71-4.94, $p<0.001$ ), and with higher scores of ECOG (OR, 1.46, $95 \% \mathrm{CI}$, $1.17-1.82, p=0.001$ ) were more likely to have willingness to designate ADs. The factors that not emerged in binary logistic regression analysis as associated with the willing to designate ADs were marital status, income, health insurance, primary tumor site, metastases, and living situation (Table 4).

\section{Discussion}

From 1985 to 2005, China's market-oriented medical reform encouraged hospitals to pursue the profit in order to fill the breach of public financial subsidy [36, 37]. Consequently, the medical system did not focus on patients' autonomy. The patients also lacked of consciousness of maintaining their rights and interests. So we did not develop an $\mathrm{AD}$ at that time. But from 2006, the disease-centered health service is gradually transformed into patient-centered health service in our country [36]. And then, we started to debate ADs. Though AD was far from common in China, it has legalized in many countries [20, $38,39]$. Because of the medical system, $77.6 \%$ (408 of 526) of patients in this study disapproved ADs. Our study also showed that none of respondents knew what was an AD or completed it, and most of them had not heard of the term "advance directive." However, $46.6 \%$ (245 of 526) were
Table 3 Comparing with attitudes of ADs between the two groups

\begin{tabular}{|c|c|c|c|c|}
\hline Variables & $\begin{array}{l}\text { Disapproved } \\
\text { ADs group (\%) }\end{array}$ & $\begin{array}{l}\text { Approved } \\
\text { ADs group (\%) }\end{array}$ & $\chi^{2}$ & $p$ value \\
\hline With whom to discuss the topic & & & 180.4 & $<0.001$ \\
\hline Spouse & $174(42.6)$ & $24(20.4)$ & & \\
\hline Parent & $116(28.4)$ & $9(7.6)$ & & \\
\hline Child & $99(24.3)$ & $19(16.1)$ & & \\
\hline Oncologist or nurse & $19(4.7)$ & $66(55.9)$ & & \\
\hline When to discuss the topic & & & 4.12 & 0.127 \\
\hline Diagnosis immediately & $65(15.9)$ & $22(18.6)$ & & \\
\hline Cancer advanced & $180(44.1)$ & $61(51.7)$ & & \\
\hline Terminally ill & $163(40.0)$ & $35(29.7)$ & & \\
\hline Where to discuss the topic & & & 244.1 & $<0.001$ \\
\hline Home & $228(55.9)$ & $12(10.2)$ & & \\
\hline Nursing home & $112(27.5)$ & $11(9.3)$ & & \\
\hline Hospice & $52(12.7)$ & $20(16.9)$ & & \\
\hline Cancer center & $16(3.9)$ & $75(63.6)$ & & \\
\hline What care to need & & & 18.8 & $<0.001$ \\
\hline Comfort care only & $230(56.4)$ & $91(77.1)$ & & \\
\hline Limited care & $137(33.6)$ & $25(21.2)$ & & \\
\hline All care possible & $41(10.0)$ & $2(1.7)$ & & \\
\hline Where to die & & & 2.35 & 0.309 \\
\hline Home & $297(72.8)$ & $87(73.7)$ & & \\
\hline Nursing home & $58(14.2)$ & $21(17.8)$ & & \\
\hline Hospital & $53(13.0)$ & $10(8.5)$ & & \\
\hline
\end{tabular}


Table 4 Analysis by binary logistic regression of the factors associated with willing to designate $\mathrm{ADs}$

\begin{tabular}{llll}
\hline Variables & Odds ratio & $95 \%$ CI & $p$ value \\
\hline Age & 1.04 & {$[1.02-1.07]$} & 0.001 \\
Gender & 0.55 & {$[0.35-0.88]$} & 0.013 \\
Marital status & 1.67 & {$[0.90-3.08]$} & 0.098 \\
Income & 1.09 & {$[0.83-1.43]$} & 0.514 \\
Health insurance & 1.23 & {$[0.63-2.39]$} & 0.543 \\
Education & 3.38 & {$[1.92-5.96]$} & $<0.001$ \\
Primary tumor site & 1.11 & {$[0.90-1.36]$} & 0.308 \\
Metastases & 0.91 & {$[0.57-1.45]$} & 0.705 \\
Living situation & 1.17 & {$[0.79-1.72]$} & 0.429 \\
Religiousness & 2.91 & {$[1.71-4.94]$} & $<0.001$ \\
ECOG performance status & 1.46 & {$[1.17-1.82]$} & 0.001 \\
\hline
\end{tabular}

ECOG Eastern Cooperative Oncology Group

interested in ADs and $22.4 \%$ (118 of 526) approved the ADs. Nowadays, more and more attention has been paid to the ADs in China. From 2010 to 2016, some of the National People's Congress (NPC) delegates formally submitted proposal about setting up regulation of ADs to the NPC. At 2013, the Beijing Living Will Promotion Association (BLWPA) was established as a non-governmental organization. By BLWPA's steady effort, millions of Chinese are familiar with ADs, and 10,000 Chinese complete the document of ADs in 2014 [30].

As found in previous studies, life-sustaining treatment not only exhausted patients and caregivers' body and spirits but also wasted of the country's precious health resources $[15,25$, 40-42]. In their view, the expenditures of aggressive therapy near EOL were pointless. Our finding also showed that most of the cancer patients choose comfort care or limited care instead of resuscitation, ventilation, intubation, and they thought aggressive therapy had no dignity. In addition, some studies have demonstrated that ADs were associated with less life-sustaining treatment $[2,5]$. Nicholas et al. study indicated that ADs were related to significantly lower levels of medical expenditures, higher utilization of health care resources, and higher rates of at-home deaths indirectly [15]. It is necessary to design ADs education program to spread information of ADs and death with dignity for further discussing with them.

The role of trust as a core element of shared decisionmaking is important for ADs [43]. It was very interesting that nearly half of (54 of 118) cancer patients who endorsed ADs had no harmonious family relationship, in contrast, most of (369 of 408) cancer patients who did not endorse ADs had harmonious family relationship. It was similar that the approved ADs group patients who had no harmonious family relationship were more likely to communicate with doctors or nurses in hospital, on the contrary, the approved ADs group patients who had harmonious family relationship were more likely to discuss ADs topic with their family members at home. The cancer patients trusted the family members more, if they had harmonious family relationship; the patients trusted the doctors or nurses more, if they had no harmonious family relationship. Patients prefer the person whom they trusted to participate in their medical decision-making [44, 45]. For medical staff, good communication should be made to foster patients' trust and improve the medical decisionmaking process.

The physicians are uncertain about when is the best time to talk about dying with the patients [46]. Our results indicate that most of participants chose cancer progression was the best time to discuss, and home was the best place to die. At the beginning of cancer disease, patients subconsciously feel that talking about end of life might somehow bring it about [46], so, they do not like involving ADs and they would like actively participating in medical decision. Instead, advanced cancer patients often gave up the medical decision-making about treatment regimens [47], they begin to discuss death because they have awareness that such a terminal stage is coming, this may be a good time to discuss ADs. In addition, Chinese patients prefer to die in peace at home as long as the condition is permitted, and they think facing death is like a fallen leaf at the EOL. The willing to die at home is determined by the traditional cultural background of China. Therefore, Chinese death philosophy is ingrainedly influenced by Confucianism which is the core idea of our traditional culture $[48,49]$.

In this study, cancer patients who approved an $\mathrm{AD}$ tended to be older than those who did not. This was consistent with previous studies [11, 21, 33, 35]. Usually, the older cancer patients talk about the death much more than the younger patients, and most of them desire for death with dignity, but they are not sure how to die, therefore, they willing to use ADs to put their wishes on paper. By this way, it could not bring mental burden to their family members and friends.

Our founding indicated that fewer males approved ADs than females. The result was the same as Blackmer et al. [29]; however, this was in contrast to the finding of Trarieux-Signol et al. study [25]. The reasons for this contradiction are not known, although it could be caused by different personality, different population, and different sample size. Needing much more data to clarify whether males discuss or register ADs more than females.

Cancer patients with higher education levels have significantly higher rates of approved ADs. This parallels the findings of Mahaney-Price et al. and Freer et al. [30, 33]. In our interview, participants with higher education levels accepted the usefulness of ADs, in addition, if the low-education participants internally comprehended of ADs they would also honor ADs. So the key point is to perform educational programming of ADs for cancer patients.

It appears from our study that religious patients were more likely to endorse ADs. This is similar to previous studies 
which demonstrated that religion was significantly associated with having an $\mathrm{AD}[25,50]$. Generally, the patients approve religion as an important consideration in their life [51]. The religious participants told us that manner of death and selfmedical-decision should be in accord with their religious doctrine and values. They often discussed state of illness and treatment plan while hospitalized, and they preferred to choose comfort care or limited care near the EOL because of the faith. However, in this study, the participants thought that health care providers rarely paid attention to their religious ideas.

Studies suggested quality of life played an important role in the ADs completion [52, 53]. Patients pay great concern to quality of life during making medical decision. Our logistic regression analysis suggested that higher scores of ECOG correlate with an increased rate of favored ADs. This implies that cancer patients with poorer quality of life were more likely to choose ADs. The result might reflect that those patients had a high opinion of ADs, mainly thanks to the improved quality of life they wanted.

In order to improve the cancer patients' knowledge and attitude toward ADs, health education about advance care planning should be adopted to create a trust relationship between patients and healthcare providers, and make them accept the concept that cancer is a chronic disease, then the physicians will initiate discussions of preferences and goals about patients' health care; and through the flat media and the net, we should propagandize excellently so as to draw more attention from people all over the country to concern about the $\mathrm{ADs}$, if more people debate the ADs, our government may set policy to adapt the development of society.

The present study had some limitations. First, our sample size was small, larger samples were needed to validate the conclusion. Second, we did not interview the 107 cancer patients who were not interested in the research. There may be some value information about why did they refuse to participate in our research. Third, the study from only two cancer centers may have selection bias. Multicenter studies are needed to validate the ADs preference and factors associated with willing to designate ADs among cancer patients.

In conclusion, cancer patients lack of knowledge of ADs, and none of them had an AD in China. Though only $22.4 \%$ (118 of 526) approved ADs, $46.6 \%$ (245 of 526) were interested in ADs. In this study, most of cancer patients wished to have comfort or limited care, and to die at home. The medical system, tradition culture of Confucian, and lack of knowledge made the non-approval of ADs so high in our sample. In addition, the approved ADs patients were more likely to discuss the ADs with health providers in the cancer center at the progression of cancer. In binary logistic regression analysis, the older patients, female, with higher education levels, with religions, and with higher scores of ECOG showed association with the willing to designate ADs.
Acknowledgments We thank all the persons who help us in this study. Compliance with ethical standards

Conflict of interest The authors declare that they have no competing interests.

Open Access This article is distributed under the terms of the Creative Commons Attribution-NonCommercial 4.0 International License (http://creativecommons.org/licenses/by-nc/4.0/), which permits any noncommercial use, distribution, and reproduction in any medium, provided you give appropriate credit to the original author(s) and the source, provide a link to the Creative Commons license, and indicate if changes were made.

\section{Reference}

1. Dzeng E, Colaianni A, Roland M, et al. (2015) Influence of institutional culture and policies on do-not-resuscitate decision making at the end of life. JAMA Intern Med 175:812-819

2. Silveira MJ, Kim SY, Langa KM (2010) Advance directives and outcomes of surrogate decision making before death. N Engl J Med 362:1211-1218

3. Burkle CM, Mueller PS, Swetz KM, et al. (2012) Physician perspectives and compliance with patient advance directives: the role external factors play on physician decision making. BMC Med Ethics 13:31

4. Pennec S, Monnier A, Pontone S, et al. (2012) End-of-life medical decisions in France: a death certificate follow-up survey 5 years after the 2005 act of parliament on patients' rights and end of life. BMC Palliat Care 11:25

5. Teno JM, Gruneir A, Schwartz Z, et al. (2007) Association between advance directives and quality of end-of-life care: a national study. J Am Geriatr Soc 55:189-194

6. Wiesing U, Jox RJ, Hessler HJ, et al. (2010) A new law on advance directives in Germany. J Med Ethics 36:779-783

7. Hackler C (2015) Finishing the Texas advance directives law. Am J Bioeth 15:58-60

8. Gristina GR, Martin E, Ranieri VM (2012) Regulation of advance directives in Italy: a bad law in the making. Intensive Care Med 38: $1897-1900$

9. Cui J, Zhou LJ, Zhao JJ (2008) Origination and status of living wills. Chinese Journal of Nursing 43(9):860-861

10. Gu X, Chen M, Liu M, et al (2016) End-of-life decision-making of terminally ill cancer patients in a tertiary cancer center in Shanghai, China. Support Care Cancer 24(5):2209-2215

11. Dow LA, Matsuyama RK, Ramakrishnan Vet al (2010) Paradoxes in advance care planning: the complex relationship of oncology patients, their physicians, and advance medical directives. J Clin Oncol 28:299-304

12. Fukui S, Fujita J, Tsujimura M, et al. (2011) Predictors of home death of home palliative cancer care patients: a cross-sectional nationwide survey. Int J Nurs Stud 48:1393-1400

13. Fukui S, Fukui N, Kawagoe H (2004) Predictors of place of death for Japanese patients with advanced-stage malignant disease in home care settings: a nationwide survey. Cancer 101:421-429

14. Mosher CE, Adams RN, Helft PR, et al (2016) Family caregiving challenges in advanced colorectal cancer: patient and caregiver perspectives. Support Care Cancer 24(5):2017-2024

15. Nicholas LH, Langa KM, Iwashyna TJ, et al. (2011) Regional variation in the association between advance directives and end-of-life Medicare expenditures. JAMA 306:1447-1453 
16. Molloy DW, Guyatt GH, Russo R, et al. (2000) Systematic implementation of an advance directive program in nursing homes: a randomized controlled trial. JAMA 283:1437-1444

17. Weeks WB, Kofoed LL, Wallace AE, et al. (1994) Advance directives and the cost of terminal hospitalization. Arch Intern Med 154: 2077-2083

18. Degenholtz HB, Rhee Y, Arnold RM (2004) Brief communication: the relationship between having a living will and dying in place. Ann Intern Med 141:113-117

19. Chambers CV, Diamond JJ, Perkel RL, et al. (1994) Relationship of advance directives to hospital charges in a Medicare population. Arch Intern Med 154:541-547

20. Asai A, Miura Y, Tanabe N, et al. (1998) Advance directives and other medical decisions concerning the end of life in cancer patients in Japan. Eur J Cancer 34:1582-1586

21. Tan TS, Jatoi A (2008) An update on advance directives in the medical record: findings from 1186 consecutive patients with unresectable exocrine pancreas cancer. J Gastrointest Cancer 39: $100-103$

22. Hopp FP (2000) Preferences for surrogate decision makers, informal communication, and advance directives among communitydwelling elders: results from a national study. Gerontologist 40: 449-457

23. Song J, Ratner ER, Wall MM, et al. (2010) Effect of an end-of-life planning intervention on the completion of advance directives in homeless persons: a randomized trial. Ann Intern Med 153:76-84

24. Singer PA, Thiel EC, Naylor CD, et al. (1995) Life-sustaining treatment preferences of hemodialysis patients: implications for advance directives. J Am Soc Nephrol 6:1410-1417

25. Trarieux-Signol S, Moreau S, Gourin MP, et al. (2014) Factors associated with the designation of a health care proxy and writing advance directives for patients suffering from haematological malignancies. BMC Palliat Care 13:57

26. Ewer MS, Taubert JK (1995) Advance directives in the intensive care unit of a tertiary cancer center. Cancer 76:1268-1274

27. Periyakoil VS, Neri E, Fong A, et al. (2014) Do unto others: doctors' personal end-of-life resuscitation preferences and their attitudes toward advance directives. PLoS One 9:e98246

28. Holley JL, Stackiewicz L, Dacko C, et al. (1997) Factors influencing dialysis patients' completion of advance directives. Am J Kidney Dis 30:356-360

29. Blackmer J, Ross L (2002) Awareness and use of advance directives in the spinal cord injured population. Spinal Cord 40:581-594

30. Mahaney-Price AF, Hilgeman MM, Davis LL, et al. (2014) Living will status and desire for living will help among rural Alabama veterans. Res Nurs Health 37:379-390

31. Harrington SE, Smith TJ (2008) The role of chemotherapy at the end of life: "when is enough, enough?". JAMA 299:2667-2678

32. Li YM (2014) "Advance directives" and death with dignity. Philosophical Trends 4:79-84

33. Freer JP, Eubanks M, Parker B et al (2006) Advance directives: ambulatory patients' knowledge and perspectives. Am J Med 119: 1088.e9-13

34. Go RS, Hammes BA, Lee JA, et al. (2007) Advance directives among health care professionals at a community-based cancer center. Mayo Clin Proc 82:1487-1490
35. Reilly BM, Magnussen CR, Ross J, et al. (1994) Can we talk? Inpatient discussions about advance directives in a community hospital. Attending physicians' attitudes, their inpatients' wishes, and reported experience. Arch Intern Med 154:2299-2308

36. Zhou K, Zhang X, Ding Y, et al. (2015) Inequality trends of health workforce in different stages of medical system reform (1985-2011) in China. Hum Resour Health 13:94

37. Sun Z, Wang S, Barnes SR (2016) Understanding congestion in China's medical market: an incentive structure perspective. Health Policy Plan 31:390-403

38. Evans N, Bausewein C, Menaca A, et al. (2012) A critical review of advance directives in Germany: attitudes, use and healthcare professionals' compliance. Patient Educ Couns 87:277-288

39. Emanuel LL, Barry MJ, Stoeckle JD, et al. (1991) Advance directives for medical care-a case for greater use. N Engl J Med 324: 889-895

40. Camhi SL, Mercado AF, Morrison RS, et al. (2009) Deciding in the dark: advance directives and continuation of treatment in chronic critical illness. Crit Care Med 37:919-925

41. Ramsay S (1995) UK doctors get advance-directive guidance. Lancet 345:913-914

42. Robertson GS (1995) Making an advance directive. BMJ 310:236238

43. Goff SL, Eneanya ND, Feinberg R, et al. (2015) Advance care planning: a qualitative study of dialysis patients and families. Clin J Am Soc Nephrol 10:390-400

44. Chawla N, Arora NK (2013) Why do some patients prefer to leave decisions up to the doctor: lack of self-efficacy or a matter of trust. J Cancer Surviv 7:592-601

45. Lee YY, Lin JL (2011) How much does trust really matter? A study of the longitudinal effects of trust and decision-making preferences on diabetic patient outcomes. Patient Educ Couns 85:406-412

46. Sanders J (2015) Finding the right words at the right time-highvalue advance care planning. N Engl J Med 372:598-599

47. Levinson W, Kao A, Kuby A, et al. (2005) Not all patients want to participate in decision making. A national study of public preferences. J Gen Intern Med 20:531-535

48. Guo Z (1995) Chinese Confucian culture and the medical ethical tradition. J Med Ethics 21:239-246

49. Zhang J, Yang D, Deng Y, et al. (2015) The willingness and actual situation of Chinese cancer patients and their family members participating in medical decision-making. Psychooncology 24:16631669

50. Oulton J, Rhodes SM, Howe C, et al. (2015) Advance directives for older adults in the emergency department: a systematic review. J Palliat Med 18:500-505

51. Smith AK, McCarthy EP, Paulk E, et al. (2008) Racial and ethnic differences in advance care planning among patients with cancer: impact of terminal illness acknowledgment, religiousness, and treatment preferences. J Clin Oncol 26:4131-4137

52. Knox GW (2003) Adherence to advance directives: advance directive needs to include additional elements. BMJ 327:1407

53. Martin DK, Thiel EC, Singer PA (1999) A new model of advance care planning: observations from people with HIV. Arch Intern Med 159:86-92 